\title{
ACTIVE DEBRIS REMOVAL MISSION DESIGN IN LOW EARTH ORBIT
}

\author{
Th. Martin ${ }^{1}$, E. Pérot ${ }^{2}$, M.-Ch. Desjean ${ }^{1}$, and L. Bitetti ${ }^{2}$ \\ ${ }^{1}$ CNES, French Space Agency \\ Av. E. Belin, Toulouse 31400, France \\ ${ }^{2}$ Thales C4I Systems \\ Parc Technologique du Canal \\ 3 Av. de l'Europe, Toulouse 31400, France
}

Active Debris Removal (ADR) aims at removing large sized intact objects - defunct satellites, rocket upper-stages - from space crowded regions. Why? Because they constitute the main source of the long-term debris environment deterioration caused by possible future collisions with fragments and worse still with other intact but uncontrolled objects. In order to limit the growth of the orbital debris population in the future (referred to as the Kessler syndrome), it is now highly recommended to carry out such ADR missions, together with the mitigation measures already adopted by national agencies (such as postmission disposal). At the French Space Agency, CNES, and in the frame of advanced studies, the design of such an ADR mission in Low Earth Orbit (LEO) is under evaluation. A two-step preliminary approach has been envisaged. First, a reconnaissance mission based on a small demonstrator $(\sim 500 \mathrm{~kg})$ rendezvousing with several targets (observation and in-flight qualification testing). Secondly, an ADR mission based on a larger vehicle (inherited from the Orbital Transfer Vehicle (OTV) concept) being able to capture and deorbit several preselected targets by attaching a propulsive kit to these targets. This paper presents a flight dynamics level tradeoff analysis between different vehicle and mission concepts as well as target disposal options. The delta-velocity, times, and masses required to transfer, rendezvous with targets and deorbit are assessed for some propelled systems and propellant less options. Total mass budgets are then derived for two end-to-end study cases corresponding to the reconnaissance and ADR missions mentioned above. 


\section{INTRODUCTION}

\subsection{Study Context}

This paper presents a flight dynamics study which has been carried out in the frame of the OTV study at CNES. The Automated Transfer Vehicle "Jules Verne" March 9, 2008, launch demonstrated the ability of Ariane5 to place $20 \mathrm{t}$ in LEO. This was the starting point for the general study to list the different missions that an OTV may accomplish.

Among the list of possible candidate missions, ADR revealed to be one of the most promising options and a dedicated Phase 0 study was initiated (see [1] for a thorough review on ADR technological solutions, challenging issues, CNES and industrial ongoing studies on the subject). First, a reconnaissance mission was designed to visit and visualize orbital targets using a small chemical propelled vehicle ( $\sim 500 \mathrm{~kg}$ mass) with the goal of achieving an in-flight demonstration of crucial technologies; secondly, the study pursued with an ADR mission and a larger vehicle (with mass in the range of 2000-5000 kg) delivering chemical propelled kits capable of deorbiting massive targets. A functional analysis was carried out to list all the possible concepts and systems capable to answer the requirements of such an ADR mission. Following on, other designs using electrical or passive propulsion systems are currently assessed in order to propose an alternative design to the "pure" chemical scenario. The task is not fully completed but some results are presented in order to illustrate how the ADR mission design is linked to the vehicle design showing, for example, the dependence between vehicle propulsion system and removal mission strategy.

\subsection{Global Context}

Since the beginning of space activities over five decades ago, more than 37000 man-made objects have been catalogued by the U.S. Space Surveillance Network. Nearly 14800 of those objects remain in orbit today, $70 \%$ of which are the orbital debris, $10 \%$ are the rocket bodies, and $20 \%$ are the satellites (less than $25 \%$ of which being operational). These figures do not include about 500000 objects too small to be tracked by ground radars or telescopes (smaller than $10 \mathrm{~cm}$ for LEO region or less than a few tenths of centimeters at geostationary altitude), but still large enough to pose a threat to any large space objects in orbit around the Earth (operational or not). In addition, collisions between debris objects could potentially lead to a continuously growing debris population (Kessler Syndrome), thus increasing the risk to operational satellites.

Current orbital population (as of March 1, 2011) is presented in Table 1.

For a couple of decades, the trend has been well identified that in the case where no mitigation measure would be adopted, the orbital debris population 
Table 1 March 1st 2011 orbital population ${ }^{a}$ decomposition by type and orbit

\begin{tabular}{lccc}
\hline & $\mathrm{LEO}^{b}$ & $\mathrm{MEO}^{b}$ & $\mathrm{GEO}^{b}$ \\
\hline Fragment & 8882 & 1118 & 19 \\
Satellite & $1871^{c}$ & 492 & 879 \\
Rocket body & 781 & 651 & 180 \\
\hline Total & $\mathbf{1 1 5 3 4}$ & $\mathbf{2 2 6 1}$ & $\mathbf{1 0 7 8}$ \\
\hline \multicolumn{4}{c}{${ }^{a}$ From NORAD catalogue (catalogued objects size $>10 \mathrm{~cm}$} \\
in LEO and $>$ 1 m in GEO). \\
her Eorth Orbit regime corresponds to orbits with apogee \\
height below 2000 km, Medium Earth Orbit (MEO) regime cor- \\
responds to orbits with apogee height over 2000 km but orbital \\
period under 21 h, and geostationary Earth Orbit (GEO) regime \\
corresponds to orbits having a period over 21 h. \\
${ }^{c}$ 1510 of these 1871 satellites were launched before March \\
2001 (older than 10 years).
\end{tabular}

will increase because of collisions between objects. This was the motivation for numerous mitigation measures adopted earlier by many countries to minimize the generation of debris (such as increasing the survivability of space vehicles to debris impacts, the implementation of collision avoidance and end-of-life disposal manoeuvres). This permitted to slow the growth of the orbital debris population. But recently, this population dramatically increased by almost 5000 new objects (larger than $10 \mathrm{~cm}$ in size) caused to the occurrence of two dramatic events, first the Chinese Fengyun 1C satellite intentional destruction in 2007 and secondly the unmanaged collision in 2009 between the operational Iridium 33 satellite and the derelict Cosmos 2251 satellite. As the confidence grew on the prediction models and more simulation were performed taken into account refined assumptions, it became even clearer that the adopted mitigation measures will not be sufficient to stabilize the debris population and the need for ADR emerged as the only way to answer to this issue.

\section{ACTIVE DEBRIS REMOVAL MISSION}

\subsection{Functional Analysis}

The main objective of an ADR mission is to remove one or several large derelict satellites or expended rocket upper-stages, called mission targets, from their crowded orbits in order to mitigate the risk of collision with other smaller debris, uncontrolled satellites, or rocket upper-stages or even operational satellites. 
Designing a space mission and the appropriate space vehicle in order to visit a given set of targets in LEO, capture these targets, and attach a propulsive or passive device so that these targets are transferred into the appropriate disposal orbit is a challenging engineering task to lead.

The aim of the functional analysis was to research, characterize, sort, rank, and give value to all lower-level functions and concepts that may be required in the scope of the whole ADR mission. The result is a tree of required functions for ground and space segments and a list of possible concepts and technologies that may be required to accomplish these functions.

\subsubsection{Mission and vehicle concepts}

The mission scenario may begin with the launch of either one unique chaser vehicle or several chaser vehicles. The chaser vehicle may be designed to process either one unique target or several targets in a sequential way. A multitarget chaser may consist either of a single vehicle performing all tasks required for the mission from rendezvous to deorbit (monolithic vehicle) or of a multitarget servicing cargo vehicle (kind of mother ship) delivering appropriate devices (or kits) to the targets.

The orbital range domain of the chaser vehicle is much wider than for a classical LEO satellite mission. This implies the requirement for higher adaptability of all subsystems (structure, power, thermal control) to very different altitudes and solar local hours. Original features like rendezvous, fly-around, possible capture, and deorbit of noncooperative targets also pose more stringent safety requirements for the orbit and attitude control subsystem.

In the case of a cargo vehicle delivering kits to targets, the tasks may be distributed in very different ways between the mother ship and the kits by the order of increasing autonomy implemented on-board the kits:

- the kit only realizes the delta-velocity required to transfer to disposal orbit.

The kit is attached to the target by the cargo vehicle after it has completed the transfer, rendezvous, and capture of the target with possibly prior damping of target tumbling motion. In case of a propulsive manoeuvre, the kit may also achieve attitude control to keep thrust vector aligned with velocity in the orbital frame and to cope with uncertainties in center of gravity location and disturbance thrust torques (in the case of minimal kit with no attitude control, the cargo main vehicle may spin up the composite formed by the kit and the target prior to jettisoning it so as to guarantee a minimal stability in thrust vector alignment);

- the kit is jettisoned by the cargo at target vicinity and autonomously realizes the close rendezvous, capture, and deorbit of the target. 
The cargo vehicle realizes the transfer, phasing, and far rendezvous with the target. Once it has jettisoned the kit, it can leave the next target; and

- the kit is jettisoned by the cargo on an intermediate orbit and realizes all functions required from in-plane transfer to capture and deorbit of the target. The cargo stays on this intermediate orbit, so that the natural longitude drift in ascending node created by the $\mathrm{J} 2$ perturbation allows it to jettison every kit into its dedicated orbital plane (the target one).

\subsubsection{Target disposal concepts}

There are several ways to remove a targeted debris from its congested orbit, some options requiring a physical contact while other options not. In the frame of this study, it was assumed that the removal chaser vehicle had to capture the targeted debris before to transfer it into another orbit where collision risks with other debris do not exist anymore or are drastically reduced. It is also conceivable to load the debris in a shuttle hold and bring it back on ground, but it was considered too costly an option to be given priority at this stage of the study.

Target disposal consists in either:

- a direct controlled reentry over noninhabited areas; or

- a perigee decrease allowing atmospheric reentry in limited time (at least less than 25 years) but without any control of the impact footprint; or

- transfer of target into a high enough disposal orbit so that it will not reenter the protected LEO region (minimum perigee of $2000 \mathrm{~km}$ ) before a sufficient long time.

The main issue raised by the uncontrolled reentry option is to comply with the French Space Act requiring a casualty risk lower than $10^{-4}$.

\subsubsection{Propulsion function}

The delta-velocity needs are achieved by propulsion means which may be different according to the mission stage. In this study, propulsion systems are called active or passive whether they require or not some kind of propellant (though for some passive propulsion, electric power or inert gas tank may be required). Active chemical (using solid or bi-liquid propellants) and electrical propulsion systems have been investigated as well as passive means like momentum transfer or electrodynamic tethers and aerodynamic drag enhancement systems. 


\subsection{Mission Target Selection \& Location}

Studies performed at JSC [2-4] have already permitted to rank the targets by order of precedence as defined by the product (mass $\times$ collision probability). The faster ADR missions remove the biggest objects with the highest collision risk, the better the outcome is on the orbital debris population. An important feature of the spatial distribution of these priority targets is their concentration on a small number of narrow inclination bands (nearly 10 bands between $65^{\circ}$ and $100^{\circ}$ whose range is about $1^{\circ}$ ), ranging in altitude from 600 to $1100 \mathrm{~km}$ and mainly on nearly circular orbits (eccentricity less than 0.01 ).

The same observation applies for LEO satellites and rocket bodies population tracked by the Space Surveillance Network (SSN catalogue). Figure 1 shows the

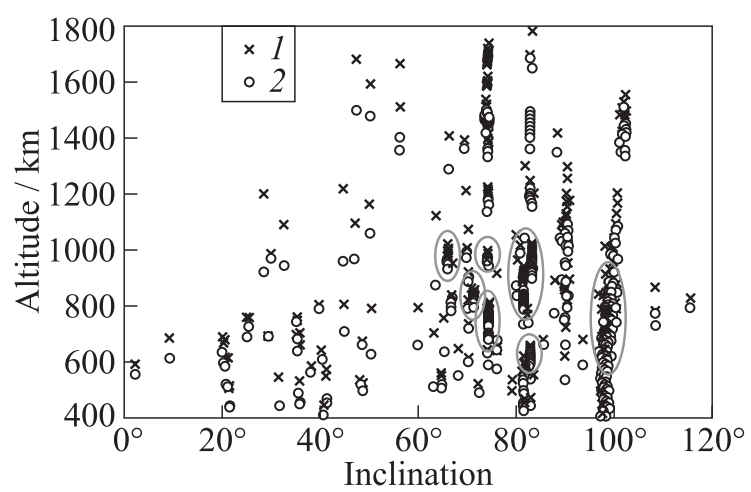

(a)

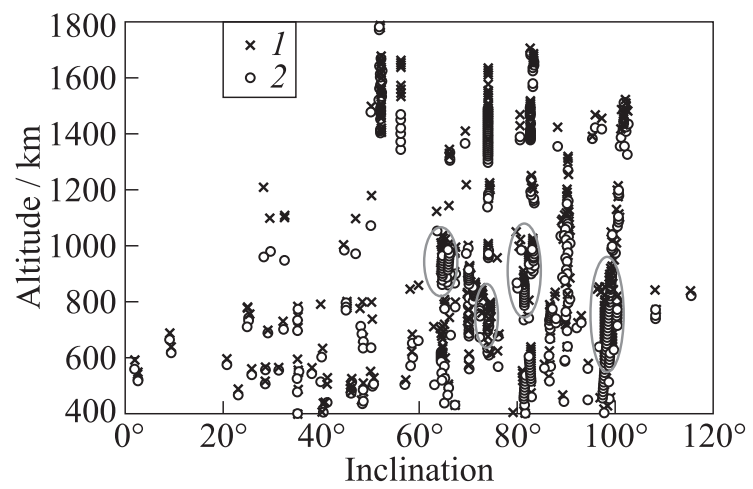

(b)

Figure 1 Rocket upper stages $(a)$ and satellites $(b)$ in LEO: 1 - apogee; and $2-$ perigee 
location in altitude and inclination of these LEO rocket bodies and satellites. The objects which belong to the priority list given in [4] have been encircled.

Note that at higher altitudes, such concentration also exists: e. g., almost 120 Russian rocket bodies $(\mathrm{R} / \mathrm{Bs})$ on $1500 \mathrm{~km} \times 74^{\circ}$ and $1400 \mathrm{~km} \times 82^{\circ}$ orbits and 50 U.S. R/Bs on $1000 \mathrm{~km} \times 90^{\circ}$ and $1500 \mathrm{~km} \times 102^{\circ}$ orbits.

At lower altitudes under $600 \mathrm{~km}$, such concentration may not be a problem since objects remain much shorter time in orbit because of higher atmospheric density (classically, a few years for objects ranging in area-over-mass ratios from 0.005 to $0.02 \mathrm{~m}^{2} / \mathrm{kg}$, compared to a few decades for altitudes between 600 and $700 \mathrm{~km}$ and several centuries at 800-kilometer altitude).

\subsection{Delta-Velocity Needs}

In the case of a single target dedicated ADR mission, the launcher may directly inject the removal tender into the target orbit. The monotarget chaser will most probably be deorbited with the target at the same time (most probably using a direct chemical propelled reentry to comply with the standard space operations policy). The delta-velocity budget calculation is then rather simple and straightforward.

In the case of a multiple targets removal mission, the second main part in the delta-velocity budget consists in the orbital transfers between targets performed by means of in-plane and out-of-plane manoeuvres, the first allowing to change orbit altitude, eccentricity, and perigee argument and to synchronize with the target anomaly, the latter allowing to change orbit inclination and right ascension of ascending node (RAAN).

In LEO regime, typical delta-velocity figures corresponding to propulsive orbital corrections are well known: about $50 \mathrm{~m} / \mathrm{s}$ to perform a 100-kilometer change in semimajor axis and about $130 \mathrm{~m} / \mathrm{s}$ to perform a one degree correction in inclination or RAAN. The mission design shall then tend to favour changes in semimajor axis instead of costly changes in inclination or RAAN:

- minimizing inclination changes is possible by designing an ADR mission sent to a specific inclination band on which the candidate targets are concentrated (see subsection 2.2); and

- avoiding RAAN changes is possible by taking advantage from the oblate Earth J2 gravitational potential perturbation that "naturally" creates a differential RAAN drift rate between orbits of different altitude and inclination.

An estimate of the cumulated delta-velocity range offered by a bi-liquid MONMMH propelled chaser vehicle, considering a wet mass of $20 \mathrm{t}$ and a dry mass of $3 \mathrm{t}$ (very optimistic assumption only for this purpose), is about $6 \mathrm{~km} / \mathrm{s}$ and 
corresponds to a cumulated change of $12,000 \mathrm{~km}$ in semimajor axis but only $50^{\circ}$ in inclination or RAAN.

Changing the orbit altitude to create the relative RAAN drift required to phase with the next target allows both to make drastic cutbacks in the global delta-velocity budget and to reach more distant RAAN (with, in return, the constraint to manage longer transfer times). The results are presented in details in subsection 3.2 .

Last point in the perspective of the end-to-end mission delta-velocity optimization is that some strategies may offer the opportunity to share the deltavelocity needed to transfer the chaser into its RAAN drift orbit towards next target and the one to transfer the target into its disposal orbit.

\subsection{Mission Duration and Optimization Problem}

The mission duration is a relevant parameter the design process has to manage. In a larger perspective, coming back to the original need for ADR missions, it is desired that 5 large targets shall be deorbited every year, but this requirement applies for the sum of ADR missions which could be planned at an international scale. At a lower scale, a European mission (processing only European targets) could last a few years.

Being given the total delta-velocity increment, the vehicle can achieve, e.g., derived from propellant tank volume and specific impulse and a list of preselected targets for the mission, the optimization problem could be posed in the simple following terms:

- finding the trajectory that maximizes the number or the total mass of removed targets (among the given list);

- satisfying time constraints (maximum duration for the mission, minimum time required for the processing of each target, maximum time allowed for transfers between targets, etc.); and

- satisfying an initial fuel mass budget.

If the vehicle mass, fuel capacity, and, therefore, delta-velocity capacity are not yet defined, it may be asked to minimize other cost criteria such as initial launch mass or overall delta-velocity. The problem may turn into a much more complex multiobjective (time and delta velocity) constrained optimization scheme to deal with.

Finally, the sensitivity of the optimized mission with respect to launch or project delays or the possibilities offered for in-flight reconfiguration of the tour caused by any contingency cases must be checked against with the vehicle design (lifetime, fuel budget). 


\section{DELTA-VELOCITY BUDGETS AND PHASES DURATIONS}

\subsection{Target Disposal Phase}

\subsubsection{Direct controlled reentry}

This is the preferred option for disposal: the object is rapidly removed from its orbit and the casualty risk is almost inexistent, the impact location being chosen over noninhabited Earth areas like South Pacific or Atlantic Oceans. Chemical propulsion with sufficient thrust force is required to perform this reentry which consists in transferring the vehicle into a 0-kilometer perigee orbit. The required delta-velocity (presented in Fig. 2) is classically performed by applying several apogee boosts ( 2 or 3 ) to gradually lower the orbit perigee altitude down to Earth surface.

In the case of solid propulsion, the delta-velocity is delivered through a single thrusters firing, which makes it difficult to control the impact footprint from higher altitudes. Using liquid propulsion makes it possible to perform several distinct boosts, allowing for a better control of the atmospheric reentry conditions (by allowing for intermediate orbit restitution) and, consequently, smaller impact footprint.

Since the final manoeuvre has to transfer the vehicle from, say, about a 200kilometer perigee orbit to a 0 -kilometer perigee orbit, the delta-velocity is about $60 \mathrm{~m} / \mathrm{s}$, and the required thrust force will depend on the thrust allotted time as well as on the initial mass to be deorbited. For a 5-minute allotted thrust time, minimum required thrust force is $200 \mathrm{~N}$ per $1 \mathrm{t}$ to be deorbited. This required thrust force varies with the inverse of the allotted thrust duration. For

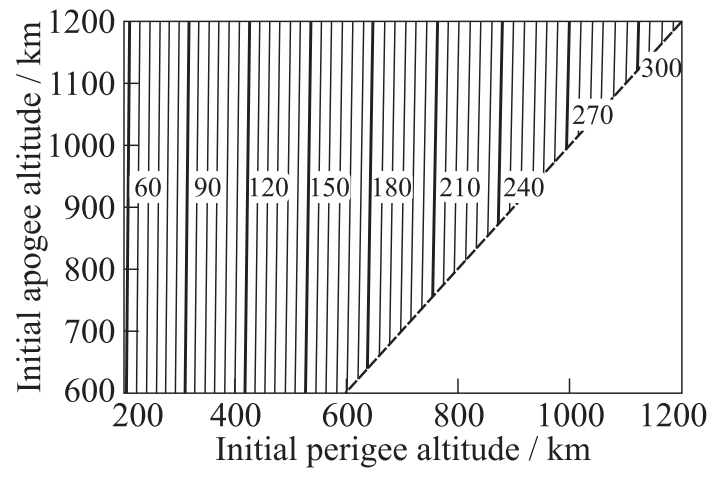

Figure 2 Delta-velocity needed for direct controlled reentry from LEO 
solid propulsion engine, the thrust time is not an issue since thrust levels are classically higher.

A spin-up or tumbling manoeuvre is classically performed, between the last deorbiting burn and Earth atmospheric interface, to remove any trajectory sensitivity to aerodynamic lift-over-drag and minimize the impact footprint.

\subsubsection{Uncontrolled reentry in a limited time}

In the case of the second disposal option which consists in lowering the perigee of the orbit so as to reach an orbit reentering the atmosphere within a limited time but in an uncontrolled manner, all active or passive propulsion means may apply. The key principle here is simply to benefit from natural forces acting on the vehicle making its orbit decaying more rapidly. Different strategies may apply to increase the orbit altitude decay (or combinations of these strategies as well) such as:

- lowering the orbit perigee altitude applying an impulsive chemical propelled boost;

- lowering the orbit altitude using electrical propulsion (leaving a nearcircular orbit, a continuous thrust will keep the orbit circular, but electrical propulsion may also perform on limited orbital arcs, e. g., sunlit part of the orbit, thereby transferring towards an eccentric orbit);

- increasing the area-over-mass ratio which dominates the aerodynamic drag force;

- using an electrodynamic tether (EDT) to create a Lorentz force induced by the motion of the tether through the Earth's magnetic field (the current carried through the tether may be naturally induced or power supplied); and

- using a momentum transfer tether connecting the chaser with the debris.

\subsubsection{Chemical Propulsion}

Figure 3 gives the perigee altitude required to limit the orbital lifetime to 25 years as a function of the initial altitude of the assumed target circular orbit and for a target area-over-mass ratio of $0.005 \mathrm{~m}^{2} / \mathrm{kg}$. Since most rocket upper stages and spacecrafts exhibit higher area-over-mass ratios (typical values ranging from 0.005 to $0.02 \mathrm{~m}^{2} / \mathrm{kg}$ ), the computed perigees (see Fig. 3), electric thrust duration (Fig. 4), and associated DeltaV (solid lines in Fig. 5) required to deorbit the target from its initial orbit may be considered as conservative values. Computations were done using the Semianalytic Tool for End of Life Analysis software 


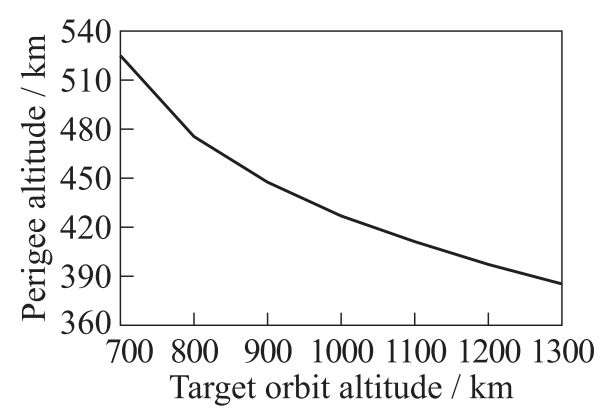

Figure 3 Maximum perigee altitude for 25-year limited orbital lifetime $(S / M$ $=0.005 \mathrm{~m}^{2} / \mathrm{kg}$ )

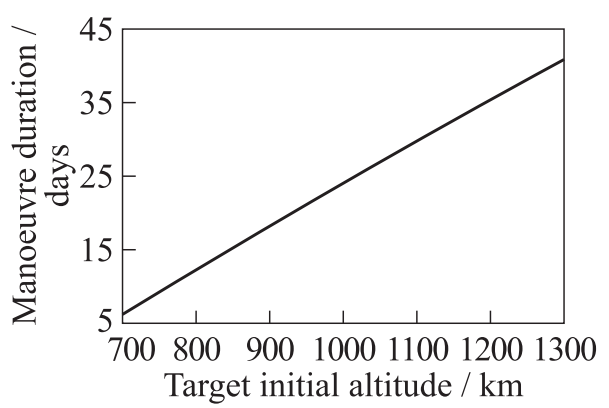

Figure 4 Time needed to reach 25year reentry orbit using electric propulsion (days per ton of deorbited mass and per $100 \mathrm{mN}$ of continuous thrust)

(STELA, http://logiciels.cnes.fr/STELA/en/logiciel.htm, see also [5]): "equivalent" constant solar flux, geomagnetic index and sphere shape drag coefficient are assumed. Drag coefficient varies with altitude $(2.1$ to 2.2 under $550 \mathrm{~km}$, rapidly increasing to 2.8 near $900 \mathrm{~km}$ ).

In comparison, with the same ballistic coefficient of $0.005 \mathrm{~m}^{2} / \mathrm{kg}$, the altitude of the initial circular orbit for which the space vehicle will deorbit in less than 25 years is about $600 \mathrm{~km}$. The delta-velocity needed to reach this circular orbit from, e.g., an initial $900 \mathrm{~km}$ circular orbit is about $160 \mathrm{~m} / \mathrm{s}$, more than $20 \%$ higher than the $125 \mathrm{~m} / \mathrm{s}$ delta-velocity needed to reach the 25-year reentering elliptical orbit (see Fig. 5).

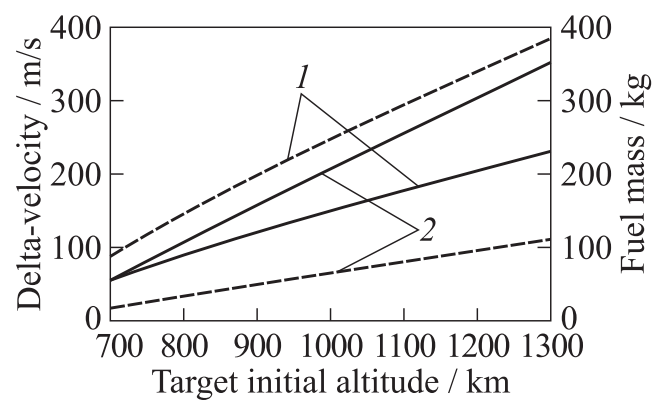

Figure 5 Compared performances between chemical (1) and electrical (2) propulsion systems for 25-year reentry 


\subsubsection{Electrical propulsion}

Fuel mass savings may be achieved using an electric propulsion system. These fuel savings are counterbalanced by a longer time required for transfer and a higher power supply demand which may require larger solar arrays, larger batteries and pose some constraints on thrust intervals (outside eclipse, may reach one third of the LEO orbital period).

Figure 4 gives the time required to transfer a $0.005 \mathrm{~m}^{2} / \mathrm{kg}$ vehicle into the 25-year circular reentry orbit (about 600-kilometer altitude) assuming continuous thrust. Time (derived here for a 1-ton vehicle and 100-millinewton thrust) increases linearly with the vehicle mass and the inverse thrust force.

Figure 5 allows to compare delta-velocities (solid lines) and fuel masses (dashed lines) between a bi-liquid chemical propulsion system $\left(I_{\mathrm{sp}}=318 \mathrm{~s}\right)$ and an electrical propulsion system $\left(I_{\mathrm{sp}}=1650 \mathrm{~s}\right)$. Continuous thrust is assumed for electrical propulsion which keeps the orbit circular and requires higher deltavelocity to reach the 25-year reentering circular orbit at about 600 -kilometer altitude. For chemical propulsion delta-velocity are lower because the reentering orbit is elliptical. Fuel masses are computed assuming a 5-ton initial mass (e.g., a 3-ton chaser deorbiting a 2-ton target).

\subsubsection{Drag augmentation system}

One way to increase natural orbit decay under drag pressure consists in attaching to the target an inflatable deorbit system providing the target with an enhanced ballistic coefficient. Nock et al. [6] presented a patented concept consisting in a relatively large, ultralightweight envelope which is stowed in a small package. One reference case they define is the deorbiting of a 1200-kilogram satellite located on a 833-kilometer sun synchronous orbit in 1 year near the solar activity peak using an envelope of $37 \mathrm{~m}$ in diameter. Another system could be similar to the IDEAS system designed for the Microscope French satellite [7].

The present authors' objective is to derive the ballistic coefficient which is required to obtain a limited orbital lifetime of 25 years for different initial orbits. The three curves represented in Fig. 6 correspond to three different initial orbits on which the inflatable device is activated: target initial circular orbit with altitude ranging from 700 to $1300 \mathrm{~km}$ (1) and two elliptical orbits with perigee altitude, respectively, 400 (2) and $600 \mathrm{~km} \mathrm{(3)} \mathrm{below} \mathrm{apogee} \mathrm{ranging} \mathrm{from} 700$ to $1300 \mathrm{~km}$. Some very small differences may appear when compared to the results presented in Fig. 3, because of the average solar flux of $130 \mathrm{sfu}$ being considered here instead of the equivalent constant solar flux proposed by STELA. A constant drag coefficient of 2.2 has also been assumed.

The curves 2 and 3 are interesting in the later perspective of using this device in addition to a chemical propelled system which would jettison the target 


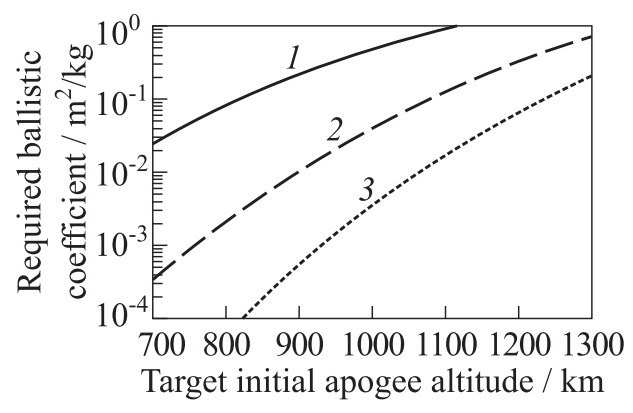

Figure 6 Required ballistic coefficient for a 25-year reentry starting from different initial orbits: circular (1) and elliptical assuming perigee altitude 400 (2) and $600 \mathrm{~km}(3)$ below apogee altitude

Table 2 Maximum 2-ton target altitudes $(\mathrm{km})$ for reentry in 25 years according to different scenarios

\begin{tabular}{lccc}
\hline \multirow{1}{*}{ Scenario } & No orbit change & \multicolumn{2}{c}{ Perigee reduction } \\
\cline { 3 - 4 } & $600 \mathrm{~km}$ & $600 \mathrm{~km}$ \\
\hline $\begin{array}{l}\text { Unchanged } S / M \\
\text { (no inflatable device) }\end{array}$ & 760 & 850 & 1020 \\
\hline $\begin{array}{l}S / M=0.05 \\
(11-\text { meter diameter envelope) }\end{array}$ & 820 & 1020 & 1180 \\
\hline $\begin{array}{l}S / M=0.1 \\
(16 \text {-meter diameter envelope) }\end{array}$ & 820 & 1230 \\
\hline
\end{tabular}

on a lower eccentric orbit. The two curves correspond to a single semimajor axis decreasing manoeuvre of 200 and $300 \mathrm{~km}$, respectively, which allows realistic lower-sized balloons and also provides the chaser with the RAAN drift rate required to meet next target in acceptable delays.

To serve as a numerical example, considering two inflatable devices consisting in small sized envelopes of $100 \mathrm{~m}^{2}$ (11-meter diameter) and $200 \mathrm{~m}^{2}$ (16-meter diameter), the maximum altitude for a 2-ton target to be removed in less than 25 years, whether this inflatable system is used or not, whether the system is combined with an initial change of the orbit or not, are derived and given in Table 2 .

\subsubsection{Electrodynamic tether}

Electrodynamic tether may allow to deorbit spacecrafts and rocket bodies from LEO. The removal concept is based on the exploitation of the Lorentz force 
caused by the interaction between an electric current flowing in a conductive tether and the Earth magnetic field. There may be two operating modes:

(1) passive: the relative motion of the conductive tether across the magnetic field lines generates an induced voltage in the tether: if the tether is put in electrical contact with the surrounding ionosphere plasma, a self-sustained current flows in the wire and a force is exerted on it according to Lorentz law. Depending on the direction of the current, this passive EDT system may provide either a propulsive thrust or a drag without the need for propellant or power supply. Such small EDT systems may be attached by a mother ship onto the target to be deorbited; and

(2) active: The removal system has a single reusable EDT system and attaches one end of the tether to the captured target. The chaser vehicle completes a round trip for each space targeted object: down to the disposal orbit with the debris, then back up to a higher orbit to continue operations. In this mode, the current is fed into the tether, against the electromotive force induced by the magnetic field, using on-board power supply. The propulsive force is exploited not only to deorbit the current target but also to raise the chaser altitude and head for the next target. This reusable EDT system is more complex, requires electrical power generation as well as current direction and intensity control, but may allow higher electrodynamic drag and shorter deorbit durations for all targeted orbits.

The passive EDT system is less efficient for high inclined orbits, where satellite velocity is nearly parallel to the magnetic field, and out-of-plane force component dominates the in-plane one, but also less efficient at high altitudes due to lower magnetic field strength and lower plasma density.

In $[8,9]$, the results based on precise numerical simulations of the tethered system are presented for the deorbiting of a 3.4-ton satellite (ADEOS) from an initial $800 \mathrm{~km}$ and $98^{\circ}$ sun synchronous orbit: 200 days only are required for reentering the atmosphere using a 10-kilometer long tether with end-mass of $50 \mathrm{~kg}$. Managing to keep the tether libration angle within acceptable limits to avoid any tumbling motion is one of the design challenging issues.

In the end, a conventional propulsion system is still required in addition to the EDT system, in order to achieve rendezvous and capture stages of the mission, because tethers are not able to provide sufficient thrust level and control for these manoeuvres.

\subsubsection{Momentum transfer tether}

Another passive concept for deorbiting a target is that of momentum transfer tether. After capture by the chaser, the tether connecting the two bodies is 


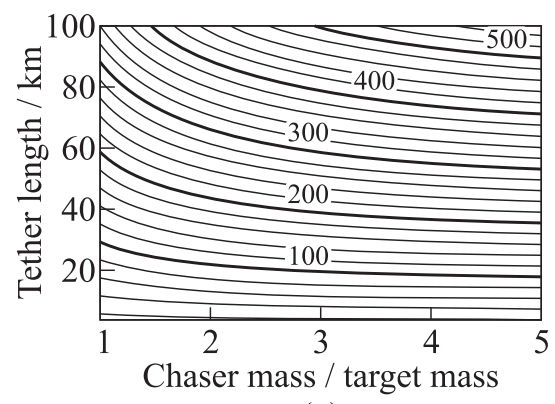

(a)

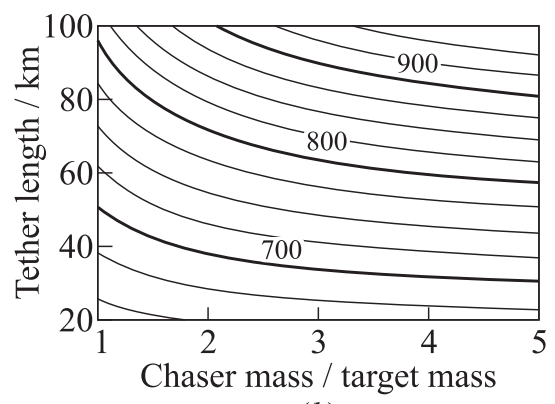

(b)

Figure 7 Target perigee decrease $(a)$ and maximum altitude for 25-year reentry using a momentum transfer tether $(b)$

unreeled with the target positioned vertically under the chaser. The upper and lower bodies are forced to orbit with the same orbital velocity than the system center of gravity, making them travelling, respectively, faster or slower than they would if they were not connected. Upon cutting of the tether, both spacecrafts are boosted into elliptical orbits where chaser apogee increases and target perigee decreases. The target may thus be transferred into a lower orbit possibly falling down in less than 25 years and the chaser altitude is raised which reduces the delta-velocity required to reach the next target.

Efficiency mainly depends on tether length and mass ratio between the two bodies as shown in Fig. $7 a$ for perigee change. Combining this perigee change with the perigee height required for 25-year reentry allows to derive the maximum target altitude till which this momentum tether system may be used (Fig. $7 b)$.

The efficiency may be increased by a factor of 2 on the perigee decrease, if a swinging motion is implied to the tether. In this case, the cutting time shall be accurately managed so that the target benefits from the maximum angular retrograde motion at the time it is released passing at the vertical under the chaser.

With this technique, it may be interesting to build a multitarget scenario in which the targets are processed in ascending order of their altitude in order to benefit from the chaser altitude increase.

\subsubsection{Reorbit beyond low Earth orbit protected region}

This third disposal option consists in transferring the debris into a disposal orbit with a perigee slightly higher than $2000 \mathrm{~km}$, so that the debris will not enter the protected LEO region for a sufficiently long time (some centuries). The delta- 


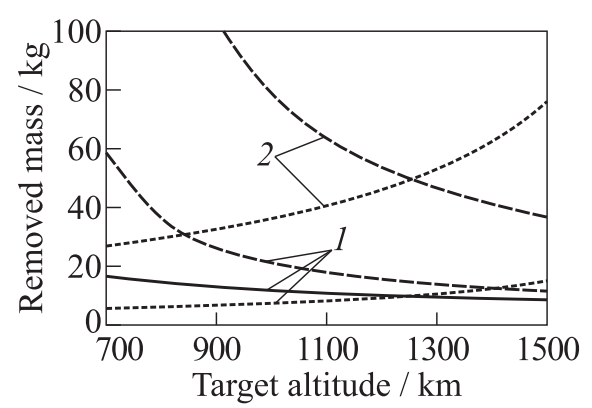

Figure 8 Removed mass per fuel kilogram used for different removal strategies and propulsion systems: 1 - chemical propulsion; and 2 - electrical propulsion velocity required to transfer into this disposal orbit from a 900-kilometer orbit is about $500 \mathrm{~m} / \mathrm{s}$ (Fig. 8). In the case of a chemical propelled system, the reorbit strategy is of low interest since the altitudes at which the deorbit cost exceeds the reorbit cost are above $1250 \mathrm{~km}$ for direct controlled reentry and $1400 \mathrm{~km}$ for 25 year reentry.

To conclude, Fig. 8 gives fuel mass efficiency for chemical and electrical propulsion systems for the different removal strategies presented in this section. Solid curve represents the direct controlled reentry solution (only achievable using chemical propulsion), dashed curves the 25-year reentry solution, and dotted curves the reorbit solution.

\subsection{Target Orbit Transfer Phase}

\subsubsection{In-plane manoeuvre costs}

Figure 9 gives the delta-velocity required to transfer using two manoeuvres from a 900-kilometer circular initial orbit to a final orbit defined by apogee altitude ranging from 600 to $2000 \mathrm{~km}$ and perigee altitude ranging from 0 to $2000 \mathrm{~km}$.

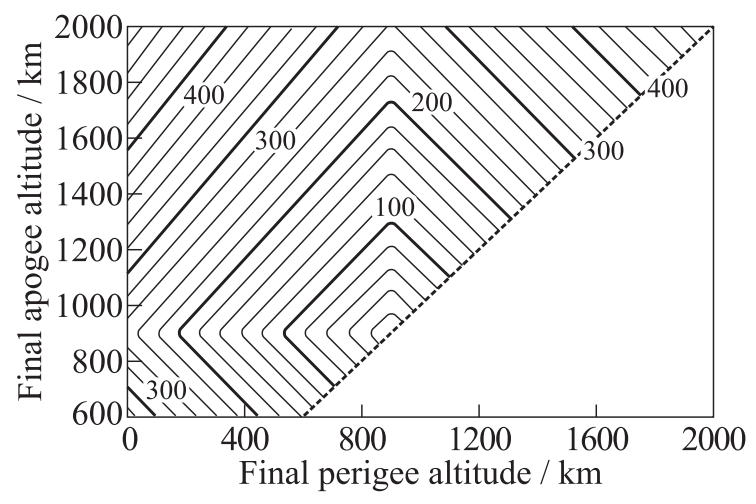

Figure 9 Delta-velocity needed to change the perigee and apogee altitudes of a 900-kilometer circular orbit 
A part of the transfer may be achieved with electrical propulsion but chemical propulsion is still required for the last rendezvous manoeuvres (homing, closing, and final approach).

\subsubsection{Out-of-plane manoeuvre costs}

Figure 10 presents the delta-velocity required to change the inclination and right ascension of the ascending node of an initial 900-kilometer circular sunsynchronous orbit.

The transfer between two targets with distant RAAN is too expensive to be achieved by means of direct out-of-plane manoeuvres. As mentioned earlier in subsection 2.3, it is more interesting to benefit from the differential RAAN drift experienced by orbits of different altitude under J2 perturbation. For nearly circular orbits (eccentricity close to 0 ), the orbital RAAN evolution under J2 perturbation depends on semimajor axis and inclination:

$$
\dot{\Omega} \approx-\frac{3}{2} R_{\text {Earth }}^{2} \sqrt{\mu} a^{-7 / 2} \cos i .
$$

Differentiating this equation according to the two orbital parameters gives the following relation:

$$
\Delta \dot{\Omega} \approx\left(-\frac{7}{2} \frac{\Delta a}{a}-\tan i \Delta i\right) \dot{\Omega} .
$$

Figure 11 gives the RAAN differential drift created by a 100-kilometer decrease of semimajor axis. For high inclination, tan $i$ becomes significant enough to allow for a coupled manoeuvre performed at orbit node and changing both

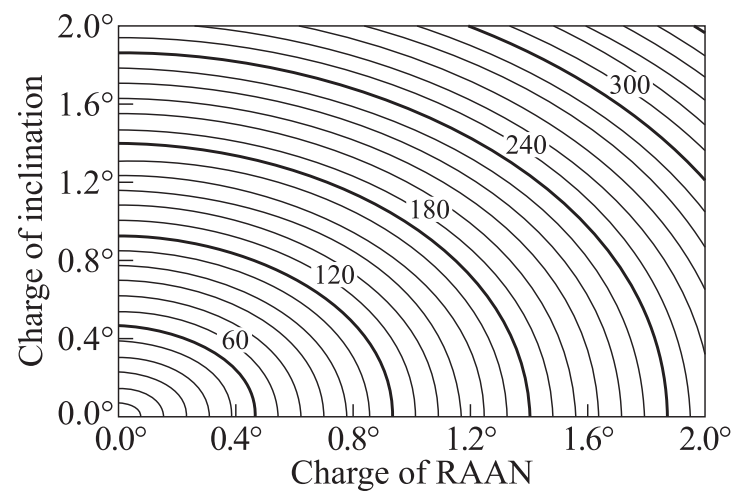

Figure 10 Delta-velocity needed to change the inclination and RAAN of a 900kilometer circular sun-synchronous orbit 


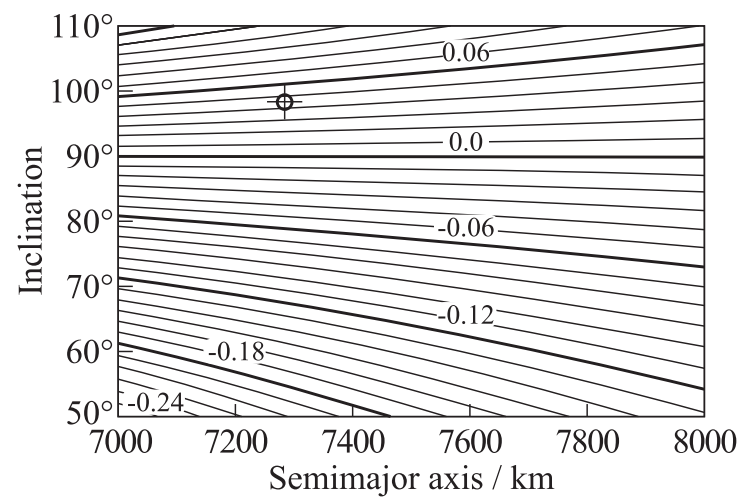

Figure 11 Differential RAAN drift created by a 100-kilometer decrease in semimajor axis

Table 3 Delta-velocity needed for a RAAN drift change of $0.1^{\circ} /$ day (using coupled manoeuvre)

\begin{tabular}{cc}
\hline Inclination & Delta-velocity, m/s \\
\hline $65^{\circ}$ & 40 \\
$75^{\circ}$ & 60 \\
$80^{\circ}$ & 80 \\
$90^{\circ}$ & 120 \\
$98^{\circ}$ & 85 \\
\hline
\end{tabular}

the semimajor axis and the inclination, which is known to be more efficient than two distinct in-plane and out-of-plane manoeuvres.

For example, considering a 900-kilometer sun-synchronous orbits $(a \sim 7280 \mathrm{~km}, \tan i$ $\sim-7, d \Omega / d t \sim 1^{\circ} /$ day), a negative change in semimajor axis of $100 \mathrm{~km}$ (cross in Fig. 11) is equivalent in terms of generated drift (about $0.045^{\circ} /$ day) to a positive change of inclination of $0.4^{\circ}$, both corrections requiring a delta-velocity manoeuvre of about $52 \mathrm{~m} / \mathrm{s}$ (see Figs. 9 and 10). If performed separately, the total delta-velocity increment required to create a differential RAAN drift of about $0.09^{\circ} /$ day is then about $104 \mathrm{~m} / \mathrm{s}$. On the contrary, if both corrections are performed simultaneously, the required delta-velocity significantly decreases at about $73 \mathrm{~m} / \mathrm{s}$.

Table 3 gives the delta-velocity required to obtain a $0.1^{\circ} /$ day RAAN drift (or $35^{\circ}$ /year) from a 1000-kilometer initial circular orbit for different inclinations.

\section{END-TO-END SCENARIOS}

This section presents the two mission design studies carried out in the frame of the OTV advanced project at the Concurrent Engineering Centre in CNES Toulouse. 


\subsection{Preparatory Mission}

The objective of such a mission would be to pave the way for future routine ADR operations by conducting flight testing and qualification of some required techniques and technologies. The vehicle would only tour among a small number of identified debris (without target capture or deorbiting) and perform some of the challenging operational phases such as:

- rendezvous phase, to demonstrate the autonomous relative navigation and final approach towards noncooperative targets; and

- inspection phase, to demonstrate formation flying with the target to characterize its surface, to estimate its attitude motion, and to identify a possible grappling point.

The mission design process consisted in:

- identifying the needs of a minimal mission visiting 4 or 5 targeted debris and aiming at demonstrating orbital transfer, rendezvous, and target inspection techniques;

- studying the feasibility of a small vehicle fulfilling to this minimal mission requirements; and

- then devising some derivative cases with increased payload, optimized propulsion, or possibility of carrying deorbiting kits.

The following description focuses on flight dynamics relevant results such as targeted population and RAAN drift waiting strategy, allotted delta-velocity and fuel mass budgets.

To illustrate the distribution of the potential targets for such a mission, let consider only European rocket bodies and satellites in sun-synchronous orbits between 700 and $1000 \mathrm{~km}$ of altitude with inclination between $97.5^{\circ}$ and $99.5^{\circ}$.

Making the assumption of constant semimajor axis and inclination, a very simple propagation over time of the right ascension of the ascending node under the sole J2 perturbation permits to have an idea of the evolution of the distribution of the target orbital planes on a 10-year horizon. Figure 12 plots the targets differential RAAN with respect to SPOT 3.

One strategy to minimize the transfer cost between two targets is to let the chaser drift in RAAN on the last visited target orbital plane until it reaches the RAAN of the following target plane. At the time when the chaser orbit RAAN coincides with the one of the next target, a correction manoeuvre is performed to change semimajor axis and inclination making the chaser stay in this new target orbital plane for rendezvous.

If delta-velocity budget and chaser fuel mass are sized assuming this strategy, then it should be investigated in which way the feasibility of this strategy is 


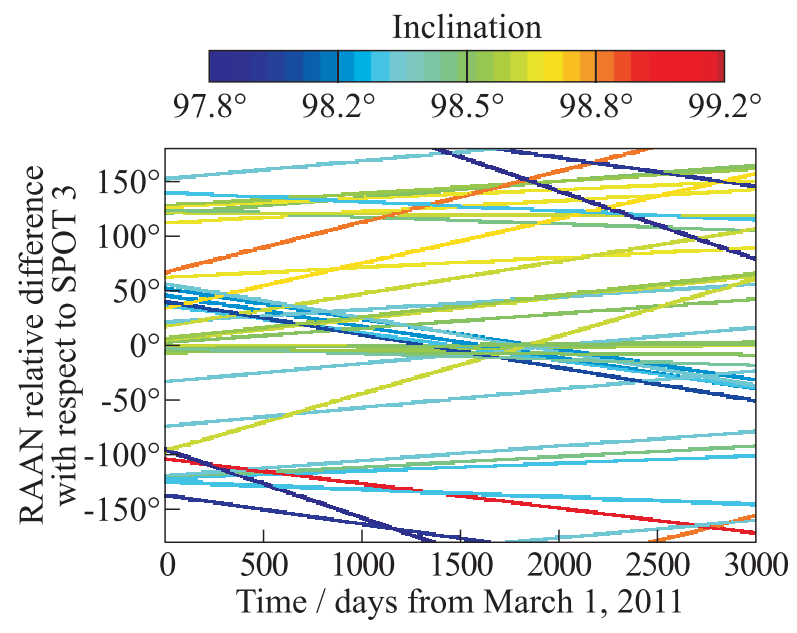

Figure 12 The RAAN distribution of 40 European rocket bodies and satellites in sun-synchronous orbit.

Table 4 Reconnaissance Mission delta-velocity budget breakdown

\begin{tabular}{|c|c|c|}
\hline Mission phase & $\begin{array}{l}\text { elta-velocity, } \\
\mathrm{m} / \mathrm{s}\end{array}$ & Number \\
\hline Rendezvous with first $t$ & 20 & 1 \\
\hline Transt & 200 & 3 \\
\hline Fly-around inspection of targ & 20 & 4 \\
\hline Direct Controlled Reentry ${ }^{d}$ & 400 & T \\
\hline Total & 1100 & \\
\hline \multicolumn{3}{|c|}{$\begin{array}{l}{ }^{a} \text { The chaser is assumed to be launched directly in the orbital plane of the first target } \\
\text { at a lower altitude (a few tens of kilometer) to permit a fast argument of latitude } \\
\text { sing. } \\
{ }^{b} \text { Required to perform inclination and in-plane correction (natural RAAN phasing } \\
\text { ategy is assumed). } \\
{ }^{c} \text { This rough estimate is intended to cover the delta-velocity required for the inspection } \\
\text { ase (no capture for this mission). } \\
{ }^{d} \text { Fifty percent margin considered wrt. the } 270 \mathrm{~m} / \mathrm{s} \text { maximum theoretical required } \\
\text { ta-velocity for 1000-kilometer altitude (see Fig. 2). }\end{array}$} \\
\hline
\end{tabular}

affected by a change in the launch date (i. e., whether it remains possible or not to find another "natural" path allowing the successive visit of a given number among the authorized targets in a limited operational time span).

Table 4 gives the allotted delta-velocity budget and its breakdown according to the different stages of the mission.

Table 5 presents the breakdown of the total mass budget according to the different subsystems of the vehicle. 
Table 5 Vehicle mass budget breakdown

\begin{tabular}{|c|c|c|c|}
\hline Subsystem of the vehicle & Mass, kg & $\begin{array}{l}\text { Mass with } \\
\text { margin, kg }\end{array}$ & $\begin{array}{c}\text { Contribution } \\
\text { in dry mass, } \\
\%\end{array}$ \\
\hline Structure & 98 & 117.6 & 36 \\
\hline Thermal control & 35 & 42 & 13 \\
\hline $\begin{array}{l}\text { Power (solar arrays, batteries, } \\
\text { PCDU, etc.) }\end{array}$ & 36.3 & 43.6 & 13 \\
\hline $\begin{array}{l}\text { AOCS (wheels, gyros, GPS, } \\
\text { SST, Mag torquer, Sun sensor, etc.) }\end{array}$ & 40 & 42 & 13 \\
\hline Data handling (OBC, mass memory) & 10.5 & 12.6 & 4 \\
\hline Communications & 9.2 & 11 & 3 \\
\hline Propulsion & 33.8 & 38.8 & 12 \\
\hline $\begin{array}{l}\text { Rendezvous and inspection module } \\
\text { (camera, lidar, radar) }\end{array}$ & 13 & 15.6 & 5 \\
\hline $\begin{array}{l}\text { Total dry mass } \\
\text { with system margin }(20 \%)\end{array}$ & 275.8 & $\begin{array}{l}323.2 \\
388\end{array}$ & 100 \\
\hline Propellant $^{b}$ & 186 & 195 & - \\
\hline Total & & 583 & \\
\hline \multicolumn{4}{|c|}{$\begin{array}{l}{ }^{a} \text { Biliquid propulsion system configuration based on two 98L MON-MMH Tanks, one } \\
\text { helium tank, eight } 10-\text {-newton thrusters }\left(I_{\mathrm{sp}}=290 \mathrm{~s} \text {, for rendezvous and inspection) and }\right. \\
\text { one } 400 \text {-newton engine }\left(I_{\mathrm{sp}}=318 \mathrm{~s} \text {, for orbital corrections and deorbiting), tubing and }\right. \\
\text { valves. } \\
{ }^{b} \text { Accounting for } 5 \% \text { of unburned propellant ( } 177 \mathrm{~kg} \text { of useful propellant), the delta- } \\
\text { velocity budget of } 1100 \mathrm{~m} / \mathrm{s} \text { is then guaranteed. }\end{array}$} \\
\hline
\end{tabular}

\subsection{Removal Mission}

The aim of the mission is to rendezvous, capture, then deorbit 5 targets of mass $1.8 \mathrm{t}$ (all assumed within a given band of inclination of less than $2^{\circ}$, at a mean altitude of $900 \mathrm{~km}$ ) using the direct controlled reentry scheme. The vehicle baseline is a mother-ship or cargo vehicle delivering kits to targets. All vehicles used chemical propulsion systems only. The mother ship is propelled by means of a bipropellant system and the deorbitation kits may use a solid or biliquid propulsion system.

Three scenarios have been defined which correspond to different hypotheses for task distribution between the mother ship and the kits (Table 6):

Scenario A corresponds to a minimalist kit with poor autonomy whose only function is to apply a single solid-propelled deorbitation boost to the target. This scenario implies that the separation between the mother ship and the target with its attached kit occurs on a low enough perigee elliptical orbit. This intermediate orbit will also be used by the mother ship to drift 
Table 6 Tasks distribution between OTV mother ship and kits

\begin{tabular}{|c|c|c|}
\hline & Mother ship & Kit \\
\hline Scenario A & $\begin{array}{l}\text { - Transfer into target orbit } \\
\text { - Rendezvous and inspection } \\
\text { - Capture } \\
\text { - Kit delivering } \\
\text { - Transfer into lower altitude } \\
\text { elliptical drift orbit } \\
\text { - Spinup and jettison of } \\
\text { kit }+ \text { target } \\
\text { - Wait for RAAN crossing } \\
\text { with next target plane or self } \\
\text { deorbitation }\end{array}$ & $\begin{array}{l}\text { - Direct reentry } \\
\text { - One single perigee lowering } \\
\text { boost } \\
\text { - Solid propulsion } \\
\text { - Gyroscopic rigidity is as- } \\
\text { sumed thanks to the prior } \\
\text { spunup of the kit }\end{array}$ \\
\hline Scenario B & $\begin{array}{l}\text { - Transfer into target orbit } \\
\text { - Rendezvous and inspection } \\
\text { - Capture } \\
\text { - Kit delivering on target and } \\
\text { jettisoning } \\
\text { - Transfer into drift orbit to- } \\
\text { wards next target or self- } \\
\text { deorbitation }\end{array}$ & $\begin{array}{l}\text { - Direct controlled reentry } \\
\text { - Two or three perigee lower- } \\
\text { ing manoeuvres } \\
\text { - Biliquid propulsion system }\end{array}$ \\
\hline Scenario C & $\begin{array}{l}\text { - Transfer into target orbit } \\
\text { - In-plane phasing and kit jet- } \\
\text { tisoning at vicinity of the } \\
\text { target } \\
\text { - Transfer on drift orbit to- } \\
\text { wards next target or self- } \\
\text { deorbitation }\end{array}$ & $\begin{array}{l}\text { - Autonomous rendezvous } \\
\text { - Inspection and capture } \\
\text { - Direct controlled reentry } \\
\text { - Two or three perigee lower- } \\
\text { ing manoeuvres } \\
\text { - Biliquid propulsion system }\end{array}$ \\
\hline
\end{tabular}

towards the next target. The chaser thus carries out intertarget transfers, rendezvous, and captures, attaches a kit to each target, then brings down the target to a lower orbit from which the kit achieves the direct reentry. The created differential RAAN drift direction is always the same: positive or negative depending on targets inclination higher or lower than $90^{\circ}$;

Scenario B corresponds to a chaser mother ship catching the different targets, attaching a biliquid propelled kit to them, then jettisoning the kit and tar- 
get and travelling to the next target. The kit achieves the direct controlled reentry of the target from its initial orbit altitude by performing several perigee lowering boosts; and

Scenario C: the mother ship transfers to the targets and jettisons autonomous biliquid propelled kits in the vicinity of targets. Then each kit achieves autonomous rendezvous, docks with and propels its target into a direct controlled reentry. The mother ship is free to travel towards the next target after kit jettison.

Detailed Assumptions. Assumptions made for the mother ship:

- dry mass ranging from 2 to 5 t;

- four 400-newton apogee boost engines and sixteen 22-newton thrusters, $I_{\mathrm{sp}}=318 \mathrm{~s}$;

- for Scenario A, two elliptical drift orbits are considered: A with perigee $400 \mathrm{~km}$ and $\mathrm{A}^{\prime}$ with perigee $200 \mathrm{~km}$ and total inclination change of $4^{\circ *}$;

- for Scenarios B and C, a fixed semimajor axis change of $\pm 200 \mathrm{~km}$ to transfer into drift orbit and total inclination change of $2^{\circ}$ for target orbit transfer*; and

- direct controlled reentry at the end of the mission from the last kit jettisoning orbit (scenario $\mathrm{A}$ and $\mathrm{A}^{\prime}$ ) or from the final target orbit (scenarios $\mathrm{B} \& \mathrm{C})$.

Assumptions made for kits in Scenarios $\mathrm{A}$ and $\mathrm{A}^{\prime}$ are:

- dry mass of $150 \mathrm{~kg}$; and

- direct reentry delta-velocity according to drift orbit perigee (see Fig. 2) achieved by solid propulsion (similar to ATK Star 17A, 16-kilonewton booster, $\left.I_{\mathrm{sp}}=286 \mathrm{~s}\right)$, with $50\left(\mathrm{~A}^{\prime}\right)$ or $100 \mathrm{~kg}(\mathrm{~A})$ of solid propellant.

Assumptions made for kits in Scenario B are:

- dry mass of $400 \mathrm{~kg}$;

- one 400-newton apogee boost engine for deorbiting, $I_{\mathrm{sp}}=318 \mathrm{~s}$; and

- eight 22-newton thrusters to allow attitude control during the target deorbit manoeuvres.

*For scenarios B\&C, the targets are processed in monotonic order of inclination, and the RAAN drift direction is optimized by increasing or decreasing the semimajor axis. Instead, for scenarios $\mathrm{A}$ and $\mathrm{A}^{\prime}$, the RAAN drift direction is constrained by the way down to the separation orbit which forces the mother ship to process targets in a monotonic order of their RAAN. This explains why a higher total inclination change was considered. 
Table 7 Expected average RAAN drift rate (deg/year) according to scenarios

\begin{tabular}{cccc}
\hline Inclination & Scenario A & Scenario $A^{\prime}$ & Scenario B or C \\
\hline $70^{\circ}$ & -104 & -152 & \pm 70 \\
$98^{\circ}$ & 42 & 62 & \pm 29 \\
\hline
\end{tabular}

Table 8 Removal Mission delta-velocity budget breakdown

\begin{tabular}{|c|c|c|c|c|}
\hline \multirow{2}{*}{$\begin{array}{l}\text { Mission } \\
\text { phase }\end{array}$} & \multicolumn{2}{|c|}{ Scenario A or $\mathrm{A}^{\prime}$} & \multicolumn{2}{|c|}{ Scenario B or C } \\
\hline & Mother ship & Kit & Mother ship & Kit \\
\hline $\begin{array}{l}\text { Transfer and far } \\
\text { range rendezvous } \\
\text { with first target }\end{array}$ & 25 & & 25 & \\
\hline Transfer to drift orbit & 130 or $180(\times 5)$ & & $102(\times 4)$ & \\
\hline $\begin{array}{l}\text { Transfer and far } \\
\text { range rendezvous } \\
\text { to subsequent targets: }\end{array}$ & $(\times 4)$ & & $(\times 4)$ & \\
\hline - in-plane & 130 or 180 & & 102 & \\
\hline - out-of-plane & 130 & & 65 & \\
\hline $\begin{array}{l}\text { Close range rendezvous, } \\
\text { inspection, and capture }\end{array}$ & & & $\begin{array}{c}20(\times 5) \\
\text { (for B only) }\end{array}$ & $\begin{array}{c}20 \\
\text { (for C only) }\end{array}$ \\
\hline $\begin{array}{l}\text { - first target } \\
\text { - subsequent targets }\end{array}$ & $20(\times 4)$ & & & \\
\hline Target direct reentry & & 120 or 60 & & 250 \\
\hline Self-direct reentry & 100 or 50 & & 250 & \\
\hline Total, $\mathrm{m} / \mathrm{s}$ & $\begin{array}{l}1920(\mathrm{~A}) \\
2320\left(\mathrm{~A}^{\prime}\right)\end{array}$ & $\begin{array}{r}120(\mathrm{~A}) \\
60\left(\mathrm{~A}^{\prime}\right) \\
\end{array}$ & $\begin{array}{l}1450(\mathrm{~B}) \\
1350(\mathrm{C})\end{array}$ & $\begin{array}{l}250(\mathrm{~B}) \\
270(\mathrm{C}) \\
\end{array}$ \\
\hline
\end{tabular}

${ }^{a}$ The mother ship is assumed to be launched directly in the orbital plane of the first target but at a lower altitude to allow fast argument of latitude phasing (typically, $50 \mathrm{~km}$ ).

${ }^{b}$ These $\Delta V$ figures are only rough order of magnitude estimates used for the purpose of this comparison between different scenarios (they correspond more to a favourable case for which the target inertial angular rate is very low (less than $0.1 \mathrm{deg} / \mathrm{s}$ ) at the time of capture.

Assumptions made for kits in scenario $\mathrm{C}$ are:

- dry mass of $500 \mathrm{~kg}$;

- one 400-newton apogee boost engine for deorbiting, $I_{\mathrm{sp}}=318 \mathrm{~s}$; and

- four 22-newton thrusters and twelve 10-newton thrusters to allow precise attitude control during rendezvous (mass of $500 \mathrm{~kg}$ ) and target deorbit manoeuvres (mass of $2.5 \mathrm{t}$ ).

Table 7 gives the expected RAAN drift rate provided by the different scenarios for two inclination cases. 
Table 9 Mass budgets with 2-/5-ton dry mass mother ship

\begin{tabular}{lccc}
\hline Case & Scenario A & Scenario B & Scenario C \\
\hline Kit & & & \\
Dry mass, kg & $150 / 15$ & $400 / 40$ & $250 / 500$ \\
Fuel mass, kg & 100 & 184 & $584 / 196$ \\
Wet mass, kg & 250 & $196 / 584$ & 696 \\
\hline 5 kits mass, kg & 1250 & 2920 & 3480 \\
\hline Mother ship & & & \\
Dry mass, kg & $2000 / 5000$ & $2000 / 5000$ & $2000 / 5000$ \\
Fuel mass, kg & $2720 / 5270$ & $2860 / 3640$ & $2820 / 3435$ \\
Wet mass, kg & $4720 / 10270$ & $3860 / 8640$ & $3820 / 8435$ \\
\hline Launch mass, kg & $5970 / 11520$ & $6780 / 11560$ & $7290 / 11915$ \\
\hline
\end{tabular}

Tables 8 and 9 give the allotted delta-velocity budget and breakdown according to the different stages of this mission (see Table 8) and the launch mass budgets for two mother ship dry mass cases (see Table 9).

\section{CONCLUDING REMARKS}

This paper presents a flight dynamics level trade-off analysis between different possible options that are candidates for ADR. Required orbital parameters and delta-velocity corrections are derived from the analysis of the debris population and the targeted objects. Several propulsion systems are assessed for both deorbit and transfer mission stages. Mass budgets are presented for three different mission scenarios using chemically propelled cargo vehicle and deorbiting kits.

However, many other issues should not be dismissed and other concepts (e.g., contactless deorbit solutions not dealt with in this paper) should be also assessed in the frame of such an ADR mission design. The first important point is that there might not exist one unique top mission scenario applying for the whole range of targeted objects and orbits. As a matter of fact, several scenarios might coexist depending on targeted objects characteristics and orbits.

As regards the atmospheric reentry of large objects, the uncontrolled reentry may not be an acceptable solution, and dedicated studies must be carried out to determine whether the casualty risk is acceptable or not. If permitted, this uncontrolled reentry scheme is likely to reveal to be a much easier option, offering many possible technological solutions and allowing also to deorbit without the need for capture (expanding foam spraying, ion beam irradiation).

Finally, as regards mission scenario definition, particular attention should be paid to the sensitivity of the mission (feasibility, time, and fuel mass required) 
with respect to a shift in the launch date. In the same way, reasonable time margins and fuel reserves should be allotted to provide for contingencies.

\section{ACKNOWLEDGMENTS}

The authors wish to thank C. Bonnal, J. M. Ruault, and P. Bultel from CNES Launcher Directorate for information and helpful guidance they provide to allow the realization of this study. They also wish to thank other members of the expert team in Toulouse, especially P. Labourdette and J. C. Berges, for the intensive and fruitful debates we had during the concurrent engineering sessions led during this study phase.

\section{REFERENCES}

1. Bonnal, C., J. M. Ruault, P. Bultel, and M. C. Desjean. High level requirements for an operational space debris deorbiter. CNES Launcher Directorate \& Toulouse Space Centre. IAC-10-A6.4.5.

2. Liou, J.-C., N. L. Johnson, and N. M. Hill. 2010. Controlling the growth of future LEO debris populations with active debris removal. Acta Astronautica 66:648-53.

3. Liou, J.-C. 2011. Active Debris Removal, a grand engineering challenge for the twenty-first century. NASA Orbital Debris Program Office. Johnson Space Center. AAS 11-254.

4. Liou, J.-C. 2011. An update on LEO environment remediation with active debris removal. Orbital Debris Quaterly News. 15(2). NASA Orbital Debris Program Office. Johnson Space Center. http://www.orbitaldebris.jsc.nasa.gov.

5. Fraysse, H., V. Morand, C. Le Fevre, F. Deleflie, S. Wailliez, A. Lamy, T. Martin, and E. Perot. Long Term Orbit Propagation Techniques developed in the frame of the French Space Act. 22nd Symposium (International) on Space Flight Dynamics. São José dos Campos, Brazil: CNES Toulouse Space Centre.

6. Nock, K. T., K. L. Gates, K. M. Aaron, and A. D. McRonald. 2010. Gossamer Orbit Lowering Device (GOLD) for safe and efficient de-orbit. AIAA Paper No. 20107824 .

7. Dupuy, C., and O. Le Couls. 2010. Gossamer technology to deorbit LEO non propulsion fitted satellite. 39th Aerospace Mechanisms Symposium Proceedings. CNES/ASTRIUM.

8. Ishige, Y., S. Kawamoto, and S. Kibe. 2004. Study on electrodynamic tether system for space debris removal. Acta Astronautica 55:917-29.

9. Kawamoto, S., T. Makida, F. Sasaki, Y. Okawa, and S. Nishida. 2006. Precise numerical simulations of electrodynamic tethers for an active debris removal system. Acta Astronautica 59:139-48. 